\title{
Back pain in a 59-year-old woman with rheumatoid arthritis
}

\author{
Roni Kraut MD, Steven Katz MD, Andrew Cave MD
}

$\mathrm{A}$

59-year-old woman presented to her family physician after one week of worsening back pain that had started shortly after an intense workout at the gym. The constant sharp pain was on the right side at the T5/T6 level. The patient had no cough, fever, shortness of breath, hemoptysis, weight loss or night sweats.

Her medical history included rheumatoid arthritis diagnosed 10 years earlier. She was receiving subcutaneous injections of methotrexate $(25 \mathrm{mg} / \mathrm{wk})$ and currently had no active joint involvement. Celiac disease had been diagnosed in 2006, and the patient was compliant with a gluten-free diet since then. She had no other medical conditions and was not taking other medications. She exercised at least three times per week and drank little alcohol. She had no history of smoking and minimal exposure to secondhand smoke. She worked as an information technology professional. Bone mineral density testing done six months previously reported a low 10-year fracture risk.

The patient appeared well, talked easily and had no difficulty breathing. Her vital signs were within normal limits. She had right- sided tenderness to palpation at the T5/T6 level on her back; there was no point tenderness on palpation of her vertebrae or ribs. Normal breath sounds were heard bilaterally on auscultation of her lungs, with no adventitious sounds. There was no pain on palpation of her calves.

We advised the patient that her pain was likely musculoskeletal in origin, secondary to her gym workout, and that it should resolve on its own. However, she returned three weeks later with worsening pain. She indicated that it was difficult to sleep and that the pain worsened on deep inspiration. Massage therapy had not helped, and she had not tried analgesics. She still had no associated symptoms, such as fever, cough, night sweats and weight loss. Upon further inquiry, we confirmed that the patient had no risk factors for pulmonary embolism. There was no change in findings on physical examination.

\section{What is the most appropriate next step?}

a. Radiography of the chest

b. Radiography of the thoracic spine

c. Radiography of the chest and D-dimer testing

d. Computed tomography of the chest

The answer is (c). Chest radiography was ordered to detect abnormalities such as infection, lung cancer and lymphoma. The Wells criteria (available at www.mdcalc.com/wells-criteria-forpulmonary-embolism-pe/) were used to evaluate the probability of a pulmonary embolism. The patient's Wells score was less than 2 (maximum 12.5), which indicated a low likelihood and suggested the need for D-dimer testing for further evaluation. ${ }^{1}$ Radiography of the thoracic spine was not ordered because a vertebral fracture was unlikely given the patient's bone mineral density and physical examination. Computed tomography (CT) was not ordered initially because chest radiography was more accessible and the Wells score was low.

A chest radiograph showed a density in the midsection of the right lung, which the radiologist's report attributed to possible scarring. The patient's D-dimer level was positive at $1.74 \mathrm{mg} / \mathrm{L}$ FEU. A CT scan for pulmonary embolism showed pleural thickening at the T5/ T6 level, with extension into the right neural foramen (Figure 1). In addition, no fractures were noted on the CT scan.

Because of the patient's worsening pain, treatment with opioids and neuropathic adjuvants was started.

\section{What should be included in the differential diagnosis?}
a. Malignant mesothelioma
b. Pleuritis secondary to rheumatoid arthritis
c. Pleural metastasis
d. All of the above

Competing interests: None declared.

This article has been peer reviewed.

The authors have obtained patient consent.

Correspondence to: Roni Kraut, rkraut@ualberta.ca

CMAJ 2016. DOI:10.1503 /cmaj.151517 
The answer is (d). Malignant mesothelioma, a neoplasm arising from the mesothelial surface of the pleural, peritoneal cavities and pericardial tissues, should be considered in the differential diagnosis. However, this diagnosis is less likely, because the patient had no known exposure to asbestos on further enquiry. Rheumatoid pleuritis is possible owing to her history of rheumatoid arthritis. Pleural metastasis of a primary tumour is also a consideration.

The patient was referred to the thoracic oncology program, and a CT-guided fine-needle biopsy was completed. The CT scan showed that the pleural thickening had not increased and had possibly decreased in size. The fine-needle biopsy sample had insufficient cells for analysis. The patient still had no cough, shortness of breath, fever, night sweats or weight loss. In consultation with the thoracic surgeon, it was felt that the pleural thickening was likely inflammatory in nature and follow-up magnetic resonance imaging (MRI) was booked for three months.

One month later, the patient had episodes of fever, muscle ache and headache. Her vital signs were normal, but there was dullness on percussion and decreased breath sounds on the right side of her chest. A chest radiograph showed a right-sided pleural effusion. A CT scan showed no change in the pleural thickening and moderate to large pleural effusion on the right side.

\section{What should be done next?}

a. Repeat fine-needle biopsy of the pleural thickening

b. Thoracentesis of pleural fluid

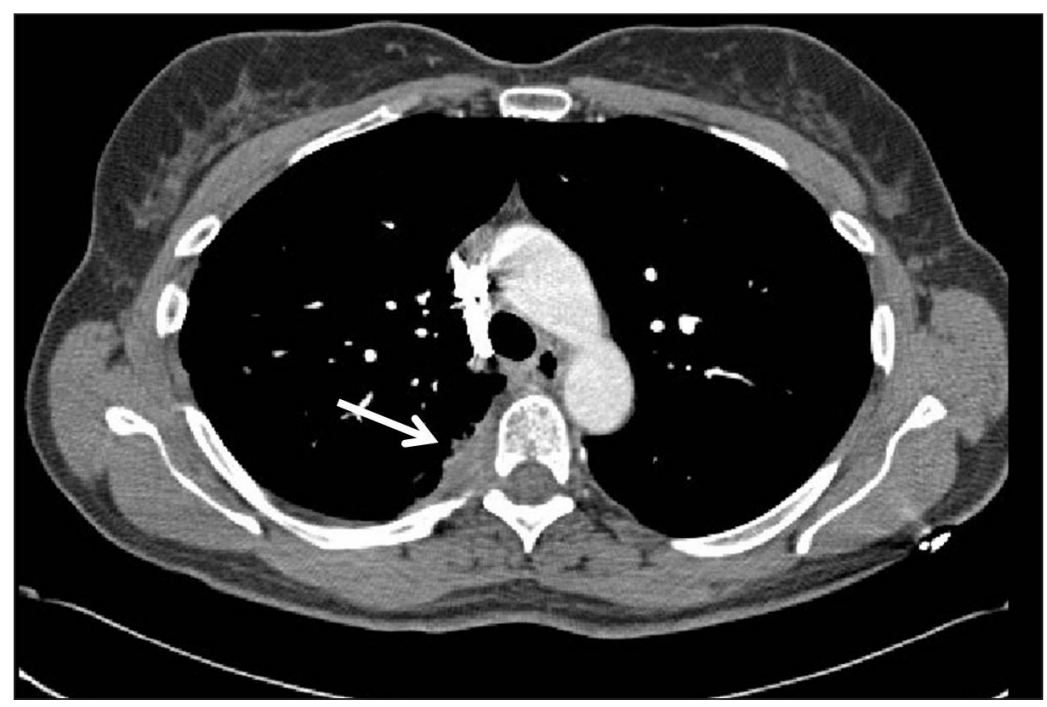

Figure 1: Computed tomography to investigate possible pulmonary embolism in a 59-year-old woman with rheumatoid arthritis who was experiencing pleuritic chest pain at the T5/T6 level. Pleural thickening is visible at the T5/T6 level, with extension into the right neural foramen (white arrow). c. Thoracoscopy with pleural biopsy

d. Expectant management and serial imaging

The answer is (b). More investigation was required given the patient's worsening symptoms, and thoracentesis was felt to be the least invasive option. The pleural fluid was negative for malignant cells. Cultures were negative, including those for tuberculosis and fungal growth. Further analysis of the pleural fluid, including cell counts and measurement of rheumatoid factor, was not done at the time of the procedure.

It was decided to proceed with a pleural biopsy because malignant disease had not been ruled out. The biopsy results showed nonspecific inflammatory changes to both the parietal and the visceral pleura that were most consistent with pleuritis secondary to rheumatoid arthritis.

In consultation with the patient's thoracic surgeon and rheumatologist, it was decided to proceed with expectant management. A follow-up MRI two months later showed that the pleural inflammation had fully resolved. The analgesics were stopped through gradual titration, and the patient continued to progress to her previous level of function.

Of note, the patient's rheumatoid arthritis temporarily worsened during this time. She noticed increasing stiffness and pain in her finger joints bilaterally about a month after the first chest CT, and her C-reactive protein level at that time was elevated. Her rheumatologist had considered adding another disease-modifying antirheumatic drug, but did not because the patient's rheumatoid arthritis symptoms subsequently improved.

\section{Discussion}

Symptomatic pleural disease secondary to rheumatoid arthritis is uncommon and typically resolves spontaneously. There have been surprisingly few studies of the prevalence of pleural involvement in rheumatoid arthritis, particularly compared with the number of reviews on the topic. Two studies reviewing $\mathrm{CT}$ findings of lung disease found the prevalence of pleural effusions to be $1.5 \%-6 \%$ among patients with rheumatoid arthritis; however, neither had a control group. ${ }^{2,3}$ A well-designed prospective case-control study in 1983 found that $11 \%$ of patients with rheumatoid arthritis had pleural thickening on examination of chest radiographs, as compared with $6 \%$ of controls; however, the group with rheumatoid arthritis did not have more respiratory symptoms. ${ }^{4}$ Ultimately, the available evidence suggests that the prevalence of pleural inflammation and effusions in rheumatoid arthritis is low, likely $6 \%$ or less, and 
that symptomatic pleural disease, as seen in our patient, is even less frequent.

Two studies found that pleural disease resolved spontaneously without treatment. In a 1967 study, 19 of 516 patients with rheumatoid arthritis had pleural effusions, which resolved within three months in 13 patients. ${ }^{5}$ Another study found that pleural effusions in all nine patients resolved within an average of 14 (range 1-36) months. ${ }^{6}$ The outcome was similar in our case, with spontaneous resolution in three months.

Evidence for treatment options remains poor. The use of nonsteroidal anti-inflammatory drugs and oral or intrapleural steroids has been described in intractable cases. ${ }^{7}$ In a minority of patients, the pleural involvement is associated with substantial morbidity and mortality. ${ }^{8}$

There is little in the literature about whether the onset of pleural disease is an indication that a patient's rheumatoid arthritis will worsen. In the 1967 study, there were 22 instances of pleural effusion; 17 occurred more than five years after the onset of rheumatoid arthritis, and in 4 of these episodes, the patient's plural effusion was accompanied by a worsening of the rheumatoid arthritis. ${ }^{5}$

Although important, analysis of the pleural fluid is neither diagnostic nor specific for rheumatoid arthritis. Typical findings include low $\mathrm{pH}$ $(<7.3)$, low glucose level $(<3.3 \mathrm{mmol} / \mathrm{L})$, elevated lactate dehydrogenase level $(>700 \mathrm{U} / \mathrm{L}$ ) and rheumatoid factor (if the rheumatoid arthritis is seropositive). ${ }^{7,9}$ The parietal pleura has been found to show characteristic features on thoracoscopic and histologic examination. ${ }^{6}$ In our patient, the tissue from the pleural biopsy was examined carefully several times, and only nonspecific inflammatory changes were found.

Pleural disease in patients with rheumatoid arthritis is uncommon and typically benign. Further investigation to support the diagnosis is often warranted, and patients should be monitored to ensure resolution.

\section{References}

1. van Belle A, Buller HR, Hulsman MV, et al. Effectiveness of managing suspected pulmonary embolism using an algorithm combining clinical probability, D-dimer testing, and computed tomography. JAMA 2006;295:172-9.

2. Cortet B, Perez T, Roux N, et al. Pulmonary function tests and high resolution computed tomography of the lungs in patient with rheumatoid arthritis. Ann Rheum Dis 1997;56:596-600.

3. Biederer J, Schnabel A, Muhle C, et al. Correlation between HRCT findings, pulmonary function tests and bronchoalveolar lavage cytology in intersitital lung disease associated with rheumatoid arthritis. Eur Radiol 2004;14:272-80.

4. Hyland RH, Gordon DA, Broder I, et al. A systematic controlled study of pulmonary abnormalities in rheumatoid arthritis. J Rheumatol 1983;10:395-405.

5. Walker WC, Wright V. Rheumatoid pleuritis. Ann Rheum Dis 1967;26:467-474.

6. Faurschou P, Francis D, Faarup P. Thoracoscopic, histological, and clinical findings in nine cases of rheumatoid pleural effusion. Thorax 1985;40:371-5.

7. Balbir-Gurman A, Yigla M, Nahir AM, et al. Rheumatoid pleural effusion. Semin Arthritis Rheum 2006;35:368-78.

8. Corcoran JP, Ahmad M, Mukherjee R, et al. Pleuro-pulmonary complications of rheumatoid arthritis. Respir Care 2014;59:e55-9.

9. Shaw M, Collins BF, Ho LA, et al. Rheumatoid arthritis-associated lung disease. Eur Respir Rev 2015;24:1-16.

Affiliation: Departments of Family Medicine (Kraut, Cave) and Medicine (Katz), University of Alberta, Edmonton, Alta.

Contributors: All of the authors contributed to the conception, preparation and editing of the manuscript, approved the version to be published and agreed to act as guarantors of the work.

CMAJ invites submissions to "What is your call?" Clinical details (including images) are presented with a multiple-choice question about the diagnosis. The answer and a brief discussion of the condition follow. We specifically invite submissions illustrating common or important radiographic and electrocardiographic diagnoses of appeal to a general audience. We require authors to obtain consent from the patient for publication of his or her story. Submit manuscripts online at http://mc.manuscript central.com/cmaj 